\title{
Cloning and Characterization of $L C B 1$, a Saccharomyces Gene Required for Biosynthesis of the Long-Chain Base Component of Sphingolipids
}

\author{
REBECCA BUEDE, CARRIE RINKER-SCHAFFER, WILLIAM J. PINTO, ROBERT L. LESTER, \\ AND ROBERT C. DICKSON* \\ Department of Biochemistry and the Lucille P. Markey Cancer Center, University of Kentucky, \\ Lexington, Kentucky 40536
}

Received 19 March 1991/Accepted 1 May 1991

\begin{abstract}
The existence of auxotrophic mutants of Saccharomyces cerevisiae having an absolute requirement for the long-chain base (lcb) component of sphingolipids suggests that sphingolipids are crucial for viability and growth. One mutant, termed the $l c b 1-1$ mutant, lacks the activity of serine palmitoyltransferase, the first enzyme in the pathway for long-chain base synthesis. Here, we present evidence that $L C B 1$ has been molecularly cloned. The size of the $L C B 1$ transcript, the direction of transcription, and transcription initiation sites were determined. In addition, the coding region and its $5^{\prime}$ and $3^{\prime}$ flanking regions were sequenced. Analysis of the DNA sequence revealed a single open reading frame of 1,674 nucleotides, encoding a predicted peptide of 558 amino acids. The hydropathy profile of the predicted peptide suggests a hydrophobic, globular, membrane-associated protein with two potential transmembrane helices. Comparison of the predicted amino acid sequence to known protein sequences revealed homology to 5 -aminolevulinic acid synthase and to 2-amino-3-ketobutyrate coenzyme A ligase. These homologies, the similarity of the chemical reactions catalyzed by the three enzymes, and the finding that $L C B 1$ restores serine palmitoyltransferase activity to an lcb1-defective strain indicate that serine palmitoyltransferase or a subunit of the enzyme is the most likely product of $L C B 1$. Homology of the LCB1 predicted protein to the Escherichia coli biotin synthetase was also observed, but the biological significance of this observation is not clear. A role for sphingolipids in sporulation is implicated by our finding that diploids homozygous for $l c b 1$ failed to sporulate.
\end{abstract}

Sphingolipids are membrane components found in animals (13), higher plants (18), and fungi (5); they are rarely present in procaryotes (20). In spite of much effort, it has been difficult to understand the exact biological role(s) of sphingolipids and their mode of action at the molecular level. In animals, sphingolipids are thought to play a role in such general cellular events as cell-to-cell recognition, regulation of cell growth, and differentiation $(13,19)$. Sphingolipids have been shown to promote a variety of specific biological activities (for a review, see reference 15). For diseases such as cancer (14), there are changes in the cellular concentration and composition of sphingolipids, but the relationship of these changes to the disease state is unclear. Recently it has been suggested that long-chain bases, such as the sphingolipid precursor sphingosine, and the breakdown products of sphingolipids, lysosphingolipids, may have important biological functions (for a review, see reference 15).

Saccharomyces cerevisiae contains a small and unique set of sphingolipids with the compositions inositol-p-ceramide, mannose-inositol- $p$-ceramide, and mannose-(inositol- $p)_{2}$-ceramide $(36,39)$. These sphingolipids and those in other fungi and plants all contain the inositol phosphorylceramide moiety and phytosphingosine. Animal sphingolipids lack inositol phosphate and instead contain oligosaccharides or phosphocholine attached to ceramide. Animal sphingolipids usually contain sphingosine instead of phytosphingosine.

To begin to understand the function(s) of sphingolipids in $S$. cerevisiae, we have begun to characterize a mutant strain blocked in sphingolipid biosynthesis (42). Strains carrying

\footnotetext{
* Corresponding author.
}

the mutant allele, $l c b 1-1$, are absolute auxotrophs and grow only when a long-chain base (lcb, phytosphingosine but not sphingosine) is added to the culture medium. The long-chain base requirement is presumably due to a block in the synthesis of phytosphingosine. In this article, we describe the isolation of the $L C B I$ gene and the determination of its DNA sequence. We also present preliminary evidence suggesting that $L C B I$ codes for serine palmitoyltransferase (3-ketosphinganine synthase [EC 2.3.1.50]), the first enzyme in the sphingolipid long-chain base biosynthetic pathway (37, 40).

\section{MATERIALS AND METHODS}

Strains and plasmids. The original $l c b$ mutant (MATa lcbl-1 inol [42]), was crossed with strain W303-1B (MATa ade2-1 canl-100 ura3-1 his3-11,15 trpl-1 leu2-3,112 [29]; obtained from R. J. Rothstein, Columbia University). Progeny from this cross were backcrossed to W303-1B, and several offspring were selected for further study, including strains X2A1B (MATa lcbl-1 ura3-1 trpl-1 his3-11,15) and 24D5 (MAT $\alpha$ lcb1-1 ura3-1 trpl-1 leu2-3,112 his3-11,15). Strain SL1 was derived from strain SJ21R (MATa ura3-52 leu2-3,112 ade1 MELI) by replacement of the $L C B 1$ allele with a mutant allele that was disrupted by inserting a $1.1-\mathrm{kb}$ URA3 DNA fragment at the SalI site of $L C B 1$. The $L C B 1:: U R A 3$-disrupted allele was prepared by transferring a 4.3-kb HindIII-StuI fragment, carrying $L C B 1$, from pLCB (Fig. 1) to pTZ18 (Pharmacia) cleaved with HindIII and SmaI. The resulting plasmid, pTZ18-LCB1 (Fig. 1), was cleaved with $S a l$ and ligated with a $1.1-\mathrm{kb} U R A 3$ DNA fragment having SalI cohesive ends (obtained from pUC- 

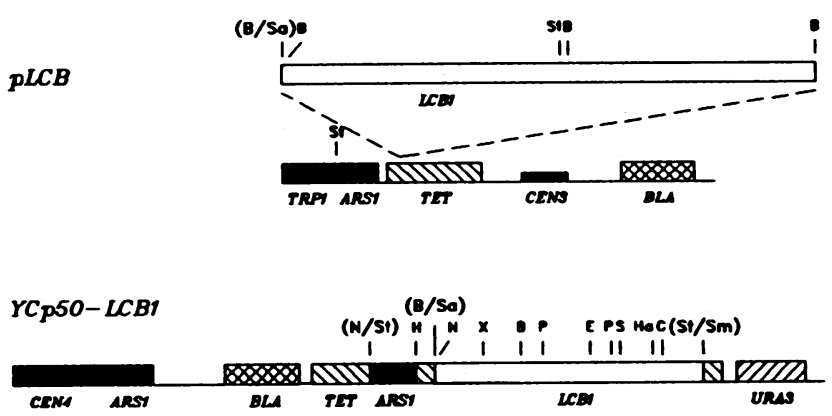

pTZ18- LCB1

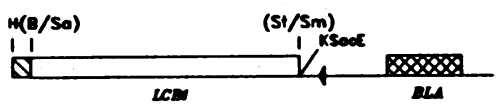

Int

FIG. 1. Structures of plasmids. The plasmid pLCB, carrying the $L C B 1$ allele, was isolated from an $S$. cerevisiae genomic library as described in the text. The approximate location of $L C B 1$ is shown. Not all restriction endonuclease sites in a given plasmid are indicated. The open arrowhead in pTZ18-LCB1 represents the T7 promoter. DNA sequences are as follows: open box, $S$. cerevisiae; TRPI and URA3, marker genes for selection in S. cerevisiae; ARSI, an $S$. cerevisiae autonomous replication sequence; $C E N 3$ and CEN4, centromere for maintenance of a single copy of the vector in yeast; BLA and TET confer ampicillin and tetracycline resistance in $E$. coli, respectively. Abbreviations for restriction endonucleases are as follows: B, BamHI; C, ClaI; E, EcoRI; H, HindIII; Ha, HpaI K, KpnI; N, NruI; P, PstI; S, SalI; Sa, Sau3A; Sac, SacI; Sm, SmaI; St, StuI; X, XbaI.

URA3 cut with SalI [44]) to yield pTZ18-LCB1::URA3. To replace the $L C B 1$ chromosomal allele with the URA3-disrupted allele, $10 \mu \mathrm{g}$ of pTZ18-LCB1::URA3 DNA was cleaved with $X b a \mathrm{I}$ and $\mathrm{ClaI}$, extracted with phenol, phenolchloroform, and chloroform, and precipitated with ethanol. The DNA was transformed into strain SJ21R with selection for $\mathrm{Ura}^{+}$transformants. Replacement of the $L C B 1$ chromosomal allele with the URA3-disrupted allele was verified by Southern blot analysis (Fig. 2). YIpLCB1-1 was constructed by inserting TRPI of $S$. cerevisiae, as a 1.4-kb HindIII fragment, into the HindIII site of pTZ18-LCB1. YIpLCB1-1 was cleaved at its unique BamHI site (Fig. 1), located on the $3^{\prime}$ side of $L C B I$, and the linear DNA was used to transform strain 24D5 with selection for $\mathrm{Ura}^{+}$transformants. Integration at the expected chromosomal location was verified by Southern blotting. Transformants were crossed to strain YPH1 (MATa ura3-52 lys2-801 ade2-101 [35]).

The plasmid pLCB (Fig. 1) was isolated from an $S$. cerevisiae genomic DNA library carried in a CEN vector. The 6.44-kb vector was pBR322 with a 0.63-kb Sau $3 \mathrm{~A} C E N 3$ DNA fragment inserted into the $P v u I I$ site of the vector and a 1.4-kb TRPI ARS1 fragment inserted into the EcoRI site of the vector. These ligations were done with molecules whose ends were made blunt ended so that the original restriction sites were destroyed. Sau3A genomic DNA fragments of 8-kb average size from strain X2180 (a/ $\alpha$ gal2/gal2) were cloned into the BamHI site of the vector (the library was a gift from ZymoGenetics, Seattle, Wash.). DNA fragments from pLCB were subcloned into YCp50 (32).

Plasmids were propagated in Escherichia coli DH5a.

Media. During the course of this work, several media were used for propagation of $l c b$-defective strains. Only the most

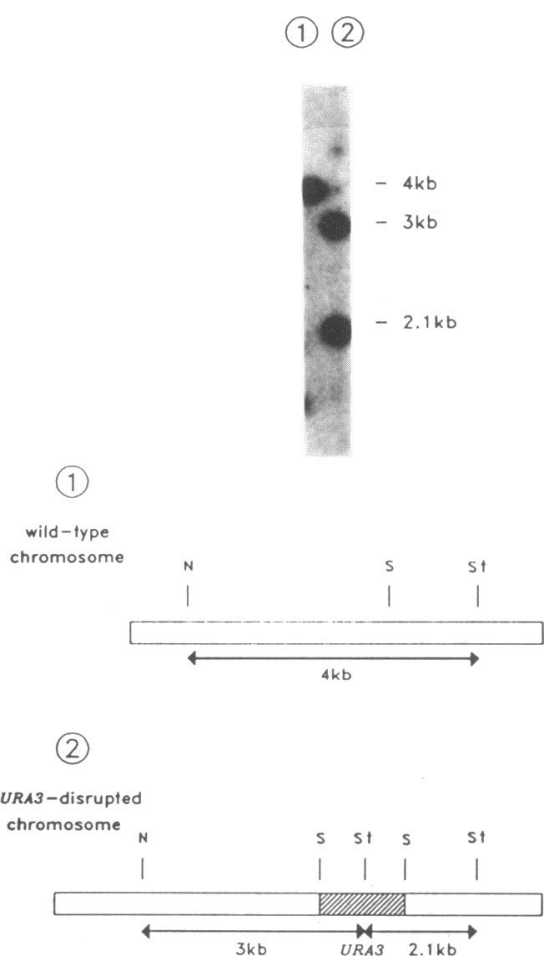

FIG. 2. Southern blot analysis of $L C B 1$. Southern blotting was used to show that the $U R A 3$ gene had disrupted the putative $L C B I$ gene at the indicated Sall site in strain SL1. Total yeast DNA was isolated and cleaved with the restriction endonucleases NruI and StuI. The cleaved DNA was subjected to Southern blot analysis. The blot was hybridized to the indicated ${ }^{32} \mathrm{P}$-labelled 4-kb $\mathrm{NruI}$ to StuI DNA fragment. Lanes: 1, DNA from the wild-type strain SJ21R; 2, DNA from the URA3-disrupted strain SL1. Abbreviations for restriction endonucleases are as follows: N, NruI; S, SalI; St, StuI.

recent versions of media are given here, as they proved most useful. PYED contained $1 \%$ peptone, $1 \%$ yeast extract, $2 \%$ agar (for plates), $50 \mathrm{mM}$ sodium succinate ( $\mathrm{pH} 5)$, inositol ( 50 $\mathrm{mg} / \mathrm{liter})$, potassium phosphate monobasic $(50 \mathrm{mg} / \mathrm{ml})$, and 2 or $4 \%$ glucose. Minimal medium contained $1 \times$ Difco yeast nitrogen base without amino acids, $50 \mathrm{mM}$ sodium succinate (pH 5), $2 \%$ glucose, $1.5 \%$ agar (for plates), inositol (50 $\mathrm{mg} /$ liter), valine (150 $\mathrm{mg} /$ liter), isoleucine $(30 \mathrm{mg} /$ liter), threonine $(200 \mathrm{mg} / \mathrm{liter})$, and the following supplements at 20 $\mathrm{mg} /$ liter: adenine sulfate, arginine- $\mathrm{HCl}$, histidine- $\mathrm{HCl}$, leucine, lysine- $\mathrm{HCl}$, methionine, tryptophan, and uracil. One or more supplements were omitted from minimal medium for selection of yeast transformants. For strains requiring longchain base, the medium was supplemented with $25 \mu \mathrm{M}$ phytosphingosine (Sigma, St. Louis, Mo.). A $10 \times$ stock solution of phytosphingosine was prepared by adding $0.25 \mathrm{ml}$ of $100 \mathrm{mM}$ phytosphingosine (dissolved in $95 \%$ ethanol) to $99.75 \mathrm{ml}$ of a $0.5 \%$ solution of tergitol (Sigma).

DNA sequencing. A 2.5-kb ClaI-XbaI DNA fragment carrying $L C B 1$ was subcloned from pTZ18-LCB1 (Fig. 1) into the same restriction sites of the M13 bacteriophage vectors pBluescript $\mathrm{KS}^{+}$and pBluescript $\mathrm{KS}^{-}$(Stratagene, La Jolla, Calif.) to yield pBluescript $\mathrm{KS}^{+}$-LCB1 and pBluescript $\mathrm{KS}^{-}$-LCB1. The $\mathrm{KS}^{+}$-LCB1 DNA was sequenced from a single-stranded template, while the $\mathrm{KS}^{-}$-LCB1 DNA was sequenced by using a double-stranded template. Synthetic 
oligonucleotide primers were used for dideoxynucleotide sequencing with Sequenase version 2.0 DNA polymerase (U.S. Biochemical, Cleveland, Ohio) essentially as recommended by the supplier.

Primer extension. The $5^{\prime}$ ends of the LCB1 mRNA were mapped by a primer extension method. A synthetic 21-base oligonucleotide, complementary to nucleotides +63 to +43 of the coding region, was used to prime the extension reaction. The primer was labelled at its $5^{\prime}$ end by using $\mathrm{T} 4$ polynucleotide kinase (Pharmacia) and $\left[\gamma^{32} \mathrm{P}\right] \mathrm{ATP}$. The extension reaction was done with avian myeloblastosis virus reverse transcriptase (Pharmacia) as described previously (7). The cDNA products of the extension reaction were separated on a $6 \%$ polyacrylamide gel containing $8 \mathrm{M}$ urea. The products of dideoxy sequencing reactions made with the same primer were used as molecular size markers.

Enzyme assays. Serine palmitoyltransferase (3-ketosphinganine synthase) assays were done by using a modification of the method of Williams et al. (43). For each assay, the following components, in a final volume of $0.2 \mathrm{ml}$, were used: $0.1 \mathrm{M} \mathrm{N}$-2-hydroxyethylpiperazine- $N^{\prime}$-2-ethanesulfonic acid (HEPES) (pH 8.3), $5 \mathrm{mM}$ dithiothreitol, $2.5 \mathrm{mM}$ EDTA ( $\mathrm{pH} 7.4$ ), $50 \mathrm{mM}$ pyridoxal phosphate, $40 \mathrm{mM}$ palmitoyl-coenzyme A (CoA), $5 \mathrm{mM}$ L-serine, $5 \mu \mathrm{Ci}$ of $\left[\mathrm{G}-{ }^{3} \mathrm{H}\right]_{\mathrm{L}-}$ serine, and $0.2 \mathrm{mg}$ of membrane protein. Each reaction was incubated with shaking for $20 \mathrm{~min}$ at $30^{\circ} \mathrm{C}$ and terminated by the addition of $0.5 \mathrm{ml}$ of $0.5 \mathrm{~N} \mathrm{NH}_{4} \mathrm{OH}$ containing $5 \mu \mathrm{mol}$ of carrier L-serine. To remove the labelled product, $3 \mathrm{ml}$ of $\mathrm{CHCl}_{3}-\mathrm{CH}_{3} \mathrm{OH}(1: 2), 50 \mu \mathrm{g}$ of 3-ketosphinganine or sphinganine (in $50 \mu \mathrm{l}$ of $95 \%$ ethanol), $2 \mathrm{ml}$ of $\mathrm{CHCl}_{3}$, and $4 \mathrm{ml}$ of $0.5 \mathrm{~N} \mathrm{NH}_{4} \mathrm{OH}$ were added with vigorous shaking. The tubes were centrifuged for $10 \mathrm{~min}$ in a clinical centrifuge, and the upper aqueous phase was removed by aspiration and discarded. Any remaining unreacted $\left[{ }^{3} \mathrm{H}\right]$ serine was removed from the $\mathrm{CHCl}_{3}$ layer by washing three times with water. A sample of the $\mathrm{CHCl}_{3}$ layer was placed in a scintillation vial and prepared for counting by evaporation over a steam bath. Four milliliters of a scintillation cocktail ( 2 liters of toluene, 1 liter of Triton $\mathrm{X}-100,350 \mathrm{ml}$ of $\mathrm{H}_{2} \mathrm{O}$ containing $0.4 \mathrm{mM}$ 2-(4'-t-butylphenyl)-5-(4'-biphenyl)-1,3,4-oxadiazole and 0.3 mM 2-(4'-biphenyl)-6-phenylbenzoxazole were added to the vial, and the radioactivity was measured in a Packard PRIAS scintillation counter. A control without enzyme was subtracted from all the assays to calculate the specific activity. One unit of enzyme activity is defined as $1 \mathrm{pmol}$ of product formed per minute.

Membranes were prepared by growing cells to mid-log phase, harvesting, resuspending in buffer $(50 \mathrm{mM}$ sodium phosphate, pH 7.0, containing $5 \mathrm{mM}$ dithiothreitol and $1 \mathrm{mM}$ phenylmethylsulfonyl fluoride), and lysing by vortexing for 3 $\min (6 \times 30 \mathrm{~s})$ with one-half volume of $0.3-$ to $0.5-\mathrm{mm}$ glass beads. Unbroken cells and debris were removed by centrifuging the homogenate at $4,000 \times g$ for $10 \mathrm{~min}$. The resulting supernatant fluid was centrifuged at $100,000 \times g$ for $1 \mathrm{~h}$ at $4^{\circ} \mathrm{C}$. The pellet was homogenized in the same buffer with a Potter-Elvehjem tissue grinder. The centrifugation and resuspension steps were repeated with the final sample resuspended in buffer containing $25 \%$ glycerol added to improve storage at $-20^{\circ} \mathrm{C}$. Protein concentration was measured by the biuret method (12) with bovine serum albumin as a standard.

Miscellaneous procedures. $S$. cerevisiae cells were transformed by the lithium acetate procedure (34). Genetic crosses and tetrad analysis were done by standard procedures (34). For Northern (RNA) blotting experiments, strain W303-1B was grown in PYED medium and total RNA was prepared by the procedure of Carlson and Botstein (6). Poly(A) ${ }^{+}$RNA was isolated by chromatography on an oligo(dT) cellulose column. Poly(A) ${ }^{+}$RNA was electrophoresed on a $1.2 \%$ agarose gel containing $0.66 \mathrm{M}$ formaldehyde and the RNA was blotted to nitrocellulose. Southern blots were done essentially as described by Maniatis et al. (22). For both blotting procedures, $\left[{ }^{32} \mathrm{P}\right] \mathrm{dATP}$-labelled probes were prepared by the method of Feinberg and Vogelstein (11).

Nucleotide sequence accession number. The Genbank number assigned to the sequence discussed herein is M63674.

\section{RESULTS}

Molecular cloning of $L C B 1$. A strain of $S$. cerevisiae requiring the long-chain base DL-erythrodihydrosphingosine was isolated previously (42) and has been shown to lack serine palmitoyltransferase activity (30). Since this strain was not suitable for molecular genetic experiments, it was crossed, as described in Materials and Methods, to give strain X2A1B $(l c b l-1)$. We isolated $L C B I$ from a genomic library by complementation of the lcbl allele. Strain X2A1B was transformed with an $S$. cerevisiae genomic library carried in a vector containing $C E N 3$ and $A R S 1$, for singlecopy propagation in yeast cells, and TRP1, for selection of $\operatorname{Trp}^{+}$yeast transformants. A genomic library carried in a single-copy vector was used because we did not know if $L C B I$ would be lethal when carried on a multicopy vector. Ten thousand $\operatorname{Trp}^{+}$transformants were selected on minimal medium plates containing phytosphingosine but lacking tryptophan. Transformants were pooled and reselected on minimal medium plates lacking both tryptophan and phytosphingosine. About one per 3,500 $\mathrm{Trp}^{+}$colonies was $\mathrm{Lcb}^{+}$.

Plasmid DNA was isolated from several $\mathrm{Lcb}^{+}$yeast transformants and transformed into $E$. coli with selection for ampicillin-resistant cells. Plasmid DNA from $E$. coli transformants was isolated and digested with restriction endonucleases. The pattern of restriction fragments indicated that the original $\mathrm{Lcb}^{+}$yeast transformants all contained the same plasmid, which carried an insert of about $8 \mathrm{~kb}$. This plasmid was called pLCB (Fig. 1).

To localize the $L C B I$ gene on the 8-kb DNA insert, we subcloned parts of the insert into the CEN4 vector YCp50 and tested the resulting plasmids for their ability to confer a $\mathrm{Lcb}^{+}$phenotype on strain X2A1B. The experiments localized $L C B 1$ to a subclone of $4.0 \mathrm{~kb}$ (Fig. 1).

Further localization of $L C B I$ was achieved by gene disruption. The 4-kb insert was disrupted at the unique $S a l$ site by insertion of the $U R A 3$ gene. The plasmid carrying the $U R A 3$ disruption was used to replace the homologous region of the chromosome in strain SJ21R as described in Materials and Methods. A potential lcbl disruption strain, SL1 (Lcb ${ }^{-}$ $\mathrm{Ura}^{+}$), was obtained. Southern blots were used to verify that the chromosome had been disrupted as expected. Total DNA isolated from SL1 and the nondisrupted parental strain SJ21R was cleaved with the restriction endonucleases $\mathrm{NruI}$ and StuI. The parental strain should show a 4-kb band on a Southern blot when the blot is probed with a ${ }^{32} \mathrm{P}$-labelled NruI-to-StuI DNA probe. Figure 2, lane 1 shows the expected radioactive band of $4 \mathrm{~kb}$. If the $U R A 3$ fragment of 1.1 $\mathrm{kb}$ is inserted into the SalI site as expected, the Southern blot of the URA3-disrupted chromosome in strain SL1 should show two bands that hybridize to the ${ }^{32} \mathrm{P}$-probe because $U R A 3$ contains a StuI cleavage site. The fragments should be 2.1 and $3 \mathrm{~kb}$ in length. The Southern blot (Fig. 2, lane 2) shows the two expected bands of hybridization 
indicating that the chromosomal allele of $L C B 1$ was disrupted by $U R A 3$ in strain SL1.

Genetic complementation analysis was used to verify that the lcb1:URA3 disruption mutation in strain SL1 was allelic to lcbl-1. Strain SL1 was crossed to strain 24D5. The resulting diploids had an $\mathrm{Lcb}^{-}$phenotype, suggesting allelism of the cloned gene and $l c b 1$. Strong support for allelism would be obtained by sporulating these diploids and showing that all tetrads give four $\mathrm{Lcb}^{-}$spores. However, such diploids failed to sporulate under a variety of conditions, suggesting that sphingolipids are needed for sporulation. An alternative genetic approach was used to demonstrate allelism. The putative $L C B I$ allele, carried on the integrating vector YIpLCB1-1, was integrated into its homologous chromosomal locus. The host strain 24D5 carried the lcb1-1 mutation. If YIpLCB1-1 did indeed carry the wild-type allele of $l c b l-1$, then the host strain should have this plasmid integrated next to $l c b l-1$. When this strain is crossed to an $L C B 1$ strain, all progeny should be $\mathrm{Lcb}^{+}$since YIpLCB1-1 would be tightly linked to $l c b l-1$. In 14 four-spored tetrads from such a cross, showing $2^{+}: 2^{-}$segregation for the Ade, Ura, and Leu phenotypes, all spores were $\mathrm{Lcb}^{+}$. We conclude that the $L C B I$ gene has been cloned.

Transcript mapping of $L C B 1$. The size of the $L C B 1$ mRNA, its location, and 5' to $3^{\prime}$ orientation were determined by Northern blotting. To determine the direction of transcription of $L C B 1$, a 4-kb DNA fragment carrying the gene was cloned into pTZ18 to yield pTZ18-LCB1 and into pTZ19 to yield pTZ19-LCB1. These plasmids were propagated in $E$. coli in the presence of a helper M13 phage in order to obtain phage particles carrying single-stranded DNA. DNA was extracted from the phage and used as a template to produce a radioactive probe. The rationale for determining the direction of $L C B 1$ transcription by using the pTZ18-LCB1 and pTZ19-LCB1 single-stranded ${ }^{32} \mathrm{P}$-labelled probes is diagrammed in Fig. 3. The rationale is based upon the probes hybridizing to opposite strands of $L C B 1$. Only one of the probes should hybridize to mRNA, and this result defines the $5^{\prime}$ to $3^{\prime}$ direction of mRNA transcription. The pTZ18LCB1 probe hybridized strongly to RNAs of 1 and $2 \mathrm{~kb}$. The pTZ19-LCB1 probe hybridized weakly to RNAs of the same size. The weak hybridization by pTZ19-LCB1 was traced to minor contamination of the probe by pTZ18-LCB1. These data indicate that $L C B 1$ is transcribed from right to left as shown in Fig. 3.

To determine whether the 1- or the 2-kb mRNA was the $L C B 1$ transcript, we used various double-stranded ${ }^{32} \mathrm{P}-\mathrm{la}-$ belled DNA fragments to probe Northern blots. The most informative probe was the 2.2-kb BamHI-to-ClaI fragment shown in Fig. 3. It hybridized only to the 2-kb mRNA. A probe to the left of the BamHI site hybridized only to the 1-kb fragment (data not shown). Since a URA3 disruption at the SalI site diagrammed in Fig. 3 created the $\mathrm{Lcb}^{-}$phenotype and since the only transcript from this region is the 2-kb transcript, we conclude that the $2-\mathrm{kb}$ transcript is the $L C B I$ mRNA.

Strains defective in $l c b 1$ lack serine palmitoyltransferase activity. To determine whether serine palmitoyltransferase activity was missing in $l c b l$-defective strains, we assayed membranes for the enzyme. The parental strain MC6A contained $54.4 \mathrm{U}$ of enzyme activity per mg of protein, while the $l c b 1$-defective strain X2A1B contained $2.5 \mathrm{U}$ per $\mathrm{mg}$ of protein, or about 20 times less enzyme activity than the parental strain: this level of activity is at the limit of detection and the actual enzyme activity may be lower. The cloned $L C B I$ allele carried in pLCB was able to restore
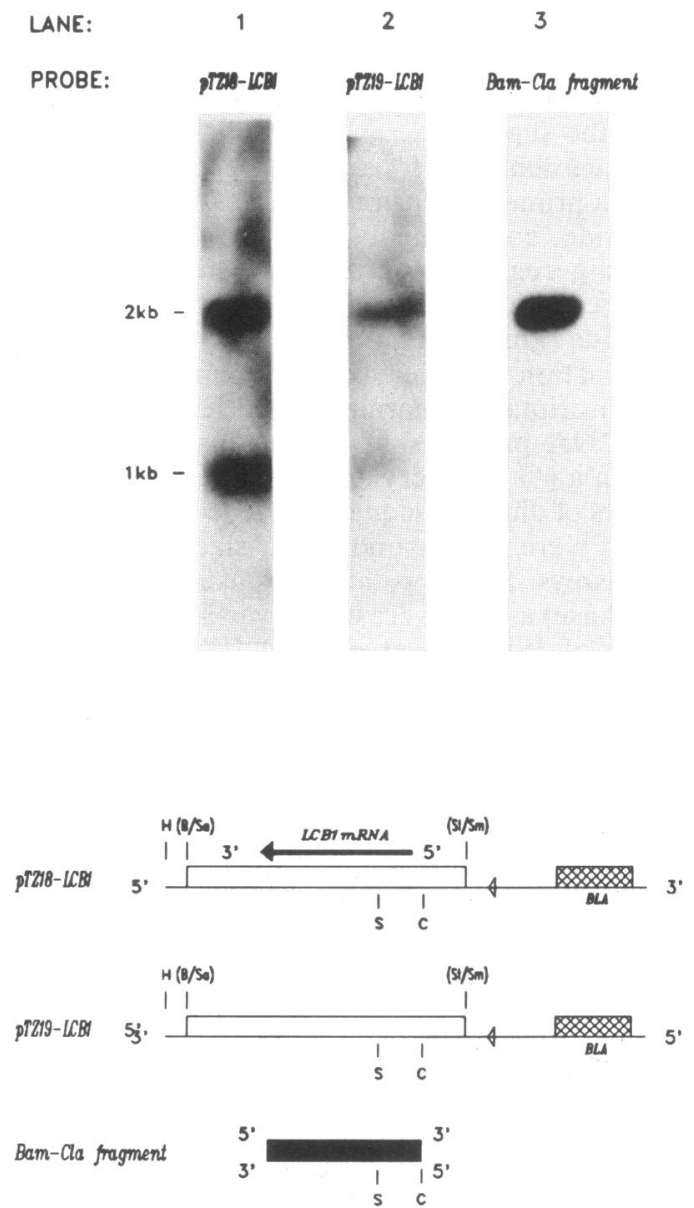

FIG. 3. Analysis of $L C B 1$ transcription. The $5^{\prime}$ to $3^{\prime}$ direction of the $L C B I$ transcript and its size were determined by electrophoresing $5 \mu \mathrm{g}$ of poly $(\mathrm{A})^{+} \mathrm{RNA}$ on an agarose gel and subjecting the gel to Northern blot analysis. The filter in lane 1 was hybridized to the single-stranded pTZ18-LCB1 probe labelled with ${ }^{32} \mathrm{P}$. The filter in lane 2 was hybridized to the single-stranded pTZ19-LCB1 probe labelled with ${ }^{32} \mathrm{P}$. The filter in lane 3 was hybridized to the doublestranded BamHI-ClaI probe labeled with ${ }^{32} \mathrm{P}$. The open arrowhead represents the T7 promoter. BLA confers ampicillin resistance in $E$. coli. $S$. cerevisiae DNA sequences are shown as open boxes, and the thin line represents pBR322 DNA. Abbreviations for restriction endonucleases are as follows: B, BamHI; H, HindIII; Sa, Sau3A; Sm, SmaI; St, StuI.

enzyme activity to about $50 \%$ of the wild-type level since three independent transformants of strain X2A1B gave 22.7, 25.6 , and $22.8 \mathrm{U}$ of enzyme activity per $\mathrm{mg}$ of protein.

DNA sequence analysis of LCB1. On the basis of the Northern blot results, the $2.5-\mathrm{kb} \mathrm{XbaI}-\mathrm{ClaI}$ fragment of DNA carrying the $L C B I$ gene was subcloned and both strands were sequenced completely. The sequence (Fig. 4) was scanned for open reading frames. A single, large open reading frame, encoding 558 amino acids and oriented in the same direction of transcription as the LCB1 mRNA, was found. This region is assumed to code for the LCB1 product because it is in the correct $5^{\prime}$ to $3^{\prime}$ orientation and because a $U R A 3$ disruption of the open reading frame at the unique SalI site created a $\mathrm{Lcb}^{-}$phenotype.

The 5' end of the LCB1 mRNA was located by primer extension analysis. The major cDNA product of the exten- 
CGC GTA TTT TTT TIT TIT TGA GGC GCC ATG ATT TCT TAC ACG GTT TCT TTT TTT TTT CCT TCT TTC CTT CTT GCT TCT CTG CTA ACA MT TIT TCA CTC ATT CTT TTT TAT AGG GGC ATA TTG CTG CGG TTA ACT GTA GTG MC GM -274 AGT MG ATT GAG AGA MA GGA MA ATA MM MA ATT CTT TTC MC ATC ATC GAG TAG CAC AGT ATA AGA GCG CTC TM CCT TCT GCC TGG CCT CCA ATA TAC
-139

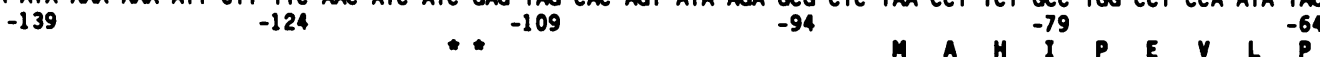
ACA TTT TGC TCG TGT AGG GTT ATT TAT CCT TTT TTC TTC CTT CCC ACC CMA MM MM MU GCA ATG GCA CAC ATC CCA GAG GTT TTA CCC

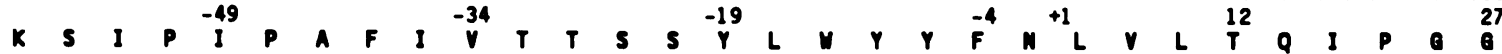
MA TCA ATA CCG ATT CCG GCA TTT ATT GTT ACC ACC TCA TCG TAC CTA TGG TAC TAC TTC AMT CTG GTG TTG ACT CM ATC CCG GGA GGC

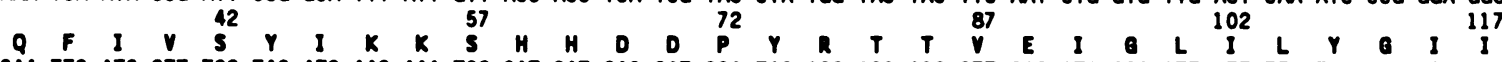
CM TTC ATC GTT TCG TAC ATC MG MM TCG CAT CAT GAC GAT CCA TAC AGG ACC ACG GTT GAG ATA GGG CTT ATT TTA TAC GGG ATC ATC

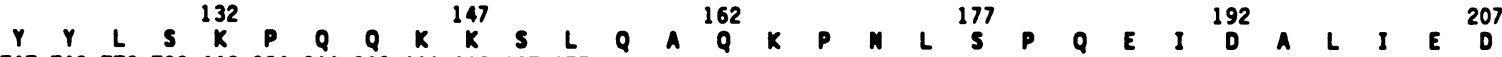
TAT TAC TTG TCC MAG CCA CM CAG MUA MAG AGT CTT CM GCA CAG $M$ AMG CCC MAC CTA TCG CCC CAG GAG ATT GAC GCG CTA ATT GAG GAC

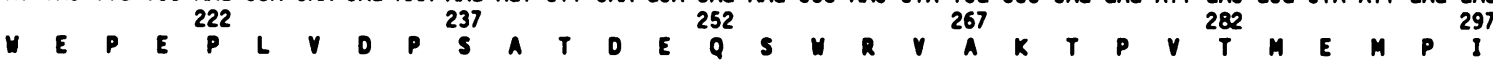
TGG GAG CCC GAG CCT CTA GTC GAC CCT TCT GCC ACC GAT GAG CM TCG TGG AGG GTG GCC AMA ACA CCC GTC ACC ATG GAM ATG CCC ATT

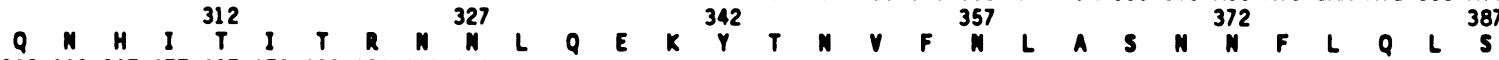
CAG MAC CAT ATT ACT ATC ACC AGA MC MC CTG CAG GAG MGG TAT ACC MT GTT TTC MT TTG GCC TCG MC MC ITT TTG CAM TTG TCC $A T T E P{ }^{402} K$ E V V GCT ACG GAG CCC GTG MM GM GTG GTC MG ACC ACT ATC MGG MT TAC GGT GTG GGC GCC TGT GGT CCC GCC GGG TTC TAC GGT MAC CAG D V H Y GAC GTT CAT TAC ACG TTG GM TAT GAT TTA GCA CAG TTC TIT GGC ACC CM GGT TCC GTT CTG TAC GGG CM GAC TIT TGT GCC GCA CCC

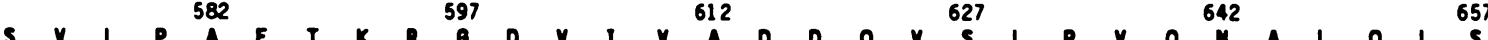
TCT GIT CTG CCT ACT TCT GTT CTG CCT GCT TTC ACA MG CGT GGT GAT GTT ATC GTG GCA GAC GAC CAG GTG TCA TTA CCA GTG CMA MAT GCT CTG CAM CTA AGC 737 732

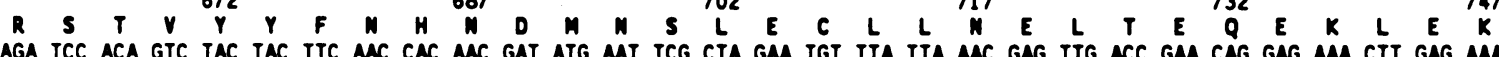
AGA TCC ACA GTC TAC TAC TIC MC CAC MAC GAT ATG MT TCG CTA GM TGT TTA TTA MAC GAG ITG ACC GM CAG GAG MM CTT GAG MM

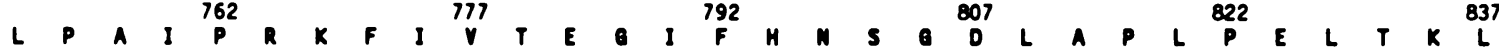
CTG CCC GCC ATT CCA AGA MAA TIT ATC GTC ACT GAG GGT ATT TTC CAC NAC TCG GGC GAT TTA GCT CCG TTG CCT GAG TTG ACT MAG CTG

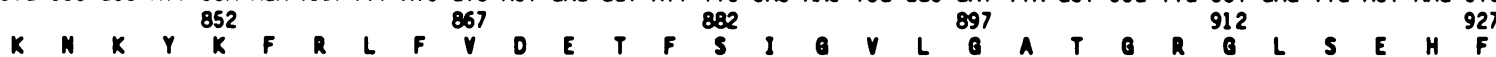
AMG MAC MG TAC MAG TTC AGA CTA ITT GIT GAC GM ACC TTC TCC ATT GGT GTT CTT GGC GCT ACG GGC CGT GGG TTG TCA GAG CAC TTC

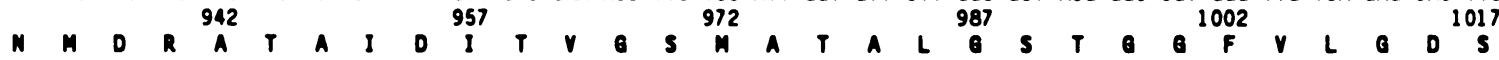
AAC ATG GAT CGC GCA ACT GCC ATT GAC ATT ACC GTT GGG TCC ATG GCC ACC GCG TTG GGG TCC ACC GGT GGT TTT GTC CTG GGT GAC AGT

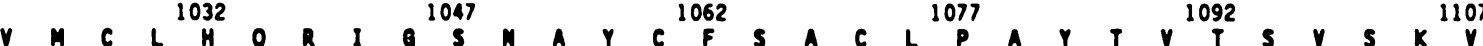
GTT ATG TGT TTG CAC CAG CGT ATT GGT TCC MIT GCA TAT TGT TIT TCT GCC TGT TTG CCG GCT TAC ACC GTC ACA TCC GTC TCC AMA GTC

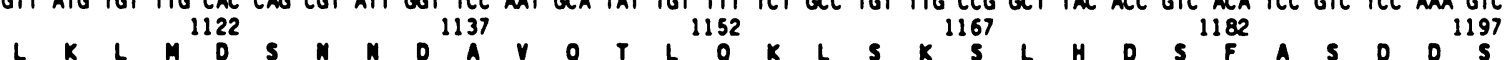

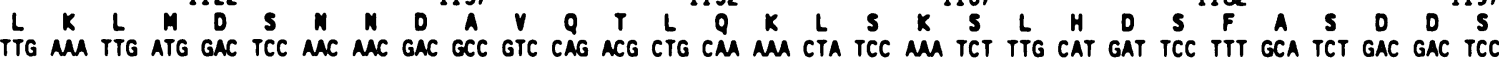

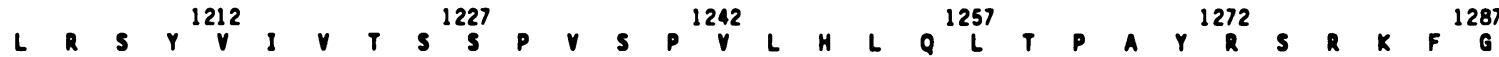
TTG CGT TCA TAC GTA ATC GTC ACG TCC TCT CCA GTG TCT CCT GTC CTA CAT CTG CM CTG ACT CCC GCA TAT AGG TCT CGC MMG TTC GGA 1302 TAC ACC TGC GM CAG CTA TTC GM ACC ATG TCA GCT TTG CM MG MAG TCC CAG ACA MC MM TTC ATT GAG CCA TAC GMA GAG GAG GAA

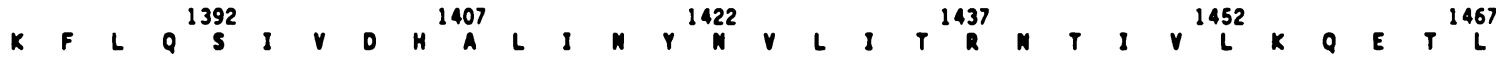
MA TTT CTG CAG TCC ATA GTA GAT CAT GCT CTT ATT MAC TAC MAC GTT CTC ATC ACA AGA AAC ACT ATT GTT TTA MAM CAG GAG ACG CTA $P \quad 1482$ CCA ATT GTC CCT AGC TTG MMA ATC TGC TGT AMC GCC GCC ATG TCC CCA GAG GM CTC MM MAT GCT TGC GM AGT GTC AAG CAG TCC ATC $L A$ A C C C Q E S M K $\begin{array}{lllll}1587 & 1602 & 1617 & 1632 & 1647\end{array}$ CTT GCC TGT TGC CMA GM TCT MT MM TM MA TAG MM GCC AGT ATA TGC ACA CGC ACA TAT ATA TAT MA TAT TTA TAC MT MAT ACA MAT MT CGT MC 1662 ATC ATC TCT GTC MM TTG ACG TGG TGC ACG GCG CCC AGA GM TGC GCT MA MT TIT CGG ATC CGA MIT ITT CTT TCC 1752
AAT MAT CGT MAC ATC ATC TCT GTC MA TTG ACG TGG TGC ACG GCG CCC AGA GM TGC GCT MA MT TIT CGG ATC CGA MAT ITT CTT TCC
18212 TTT TAC CAT CGA GGC MA GCA ACC TGT ATT ATT TAT TTG TTT-A AT TAT TMA TAG MA AGA MGG GAG TAC TTT CGT GGT ACG CTT TCT TGA gCA TTT TCG GTT TCA CTA GGC AGA GM CTA ACA CMA gAg ACA CAG CM ACA TCA MC MG GTT MA ACA GCA CAC CM GGC Mat ATG ATG CAT ITT AGA MG 1932 MA TCC AGT ATC AGT MC ACG AGT GAT CAT GAC GGA GCG MC CGT GCC TCA GAT GTC MAG ATT TCT GMA GAT GAC 2007 $\begin{array}{llll}2022 & 2067 & 2082 & 2097 \\ \text { GCA AGA TTG MG ATG CGT ACT GCT TCC GTT GCT GAT CCT A } & 2052 & & 2097\end{array}$

FIG. 4. DNA sequence of $L C B 1$. The nucleotide sequence of the $L C B I$ gene of $S$. cerevisiae is presented along with the deduced protein sequence of the 558 amino acids. The predicted translation start codon is indicated by +1 , and the end of the open reading frame is designated by an @. Asterisks above nucleotides indicate the $5^{\prime}$ ends of mRNAs as determined by primer extension analysis.

sion reaction had a $5^{\prime}$ terminus corresponding to the -25 position and a minor product corresponding to the -24 position. These $5^{\prime} \mathrm{mRNA}$ termini are indicated by asterisks along the sequence shown in Fig. 4.
Predicted amino acid sequence. The nucleotide sequence of the open reading frame was used to predict the amino acid sequence of the LCB1 peptide. The results of the prediction are illustrated above each codon of the nucleotide sequence 


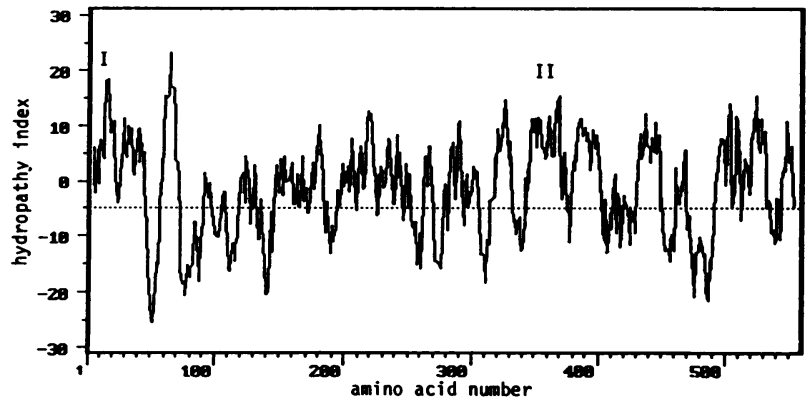

FIG. 5. Hydropathy profile of the LCB1 protein. The hydropathy of the predicted LCB1 peptide was analyzed according to the method of Kyte and Doolittle (17) by using a span setting of nine amino acid residues. Two transmembrane helices, indicated by roman numerals, were predicted by the procedure of Eisenberg et al. (10).

(Fig. 4) beginning with the first ATG codon at position +1 and ending with the stop codon TAA at position +1675 . Assuming that this ATG codon is the true translation initiation site, the product of the open reading frame is a protein of 558 amino acids with a molecular weight of 62,232 .

Because serine palmitoyltransferase activity is present in the membrane fraction of lysed cells, we expected the LCB1 protein to be membrane associated. The hydrophobicity of the deduced protein sequence was therefore examined (Fig. $5)$. The protein is moderately hydrophobic; $42.2 \%$ of the 558 amino acid residues are nonpolar, $36.9 \%$ are polar, and $20.9 \%$ are charged. According to the theory of Kyte and Doolittle (17), the grand average hydropathy score for the LCB1 product is -1.39 , a value that places the LCB1 peptide in the same class as globular proteins. A globular, rather than integral membrane, protein is also predicted by the procedure of Eisenberg et al. (10). In addition, this analysis predicts two very hydrophobic, membrane-associated helices which are designated by roman numerals in Fig. 5. Helix I spans amino acid residues 12 to 32 and has the sequence IPIPAFIVTTSSYLWYYFNLV, while helix II spans residues 344 to 373 and has the sequence ATAIDITV GSMATALGSTGGFVLG.

Residue 80 is a potential glycosylation site of the type Asn-X-Ser where the sugar would be $\mathrm{N}$ linked to the Asn residue (23).

\section{DISCUSSION}

We have described the isolation and characterization of an $S$. cerevisiae gene, termed $L C B 1$. Several results suggest that $L C B 1$ codes for serine palmitoyltransferase. The cloned gene complements a mutant lacking serine palmitoyltransferase activity, by allowing growth in the absence of exoge-

FIG. 6. Comparison of the deduced amino acid sequence of LCB1 to other proteins. The protein sequences of LCB1 and the mouse (ALSM), chicken (ALSC), and yeast (ALSY) 5-aminolevulinic acid synthases were compared by using the procedure of Pearson and Lipman (28) and aligned for maximum similarity. The 2-amino-3-ketobutyrate CoA ligase (EKBL) (1) and the biotin synthetase (EBIO) (27) sequences were identified and aligned by using the FASTA algorithm as described in the text. Colons represent identity between residues, while dots indicate conservative replacements by similar residues. Insertions made during the alignment optimization process are indicated by dashes. nous long-chain base. In addition, the cloned gene restores serine palmitoyltransferase activity to the $l c b 1-1$ mutant strain. Finally, the predicted amino acid sequence of the LCB1 protein shows high similarity to the enzymes 5-aminolevulinic acid synthase (ALA synthase) and 2-amino-3-
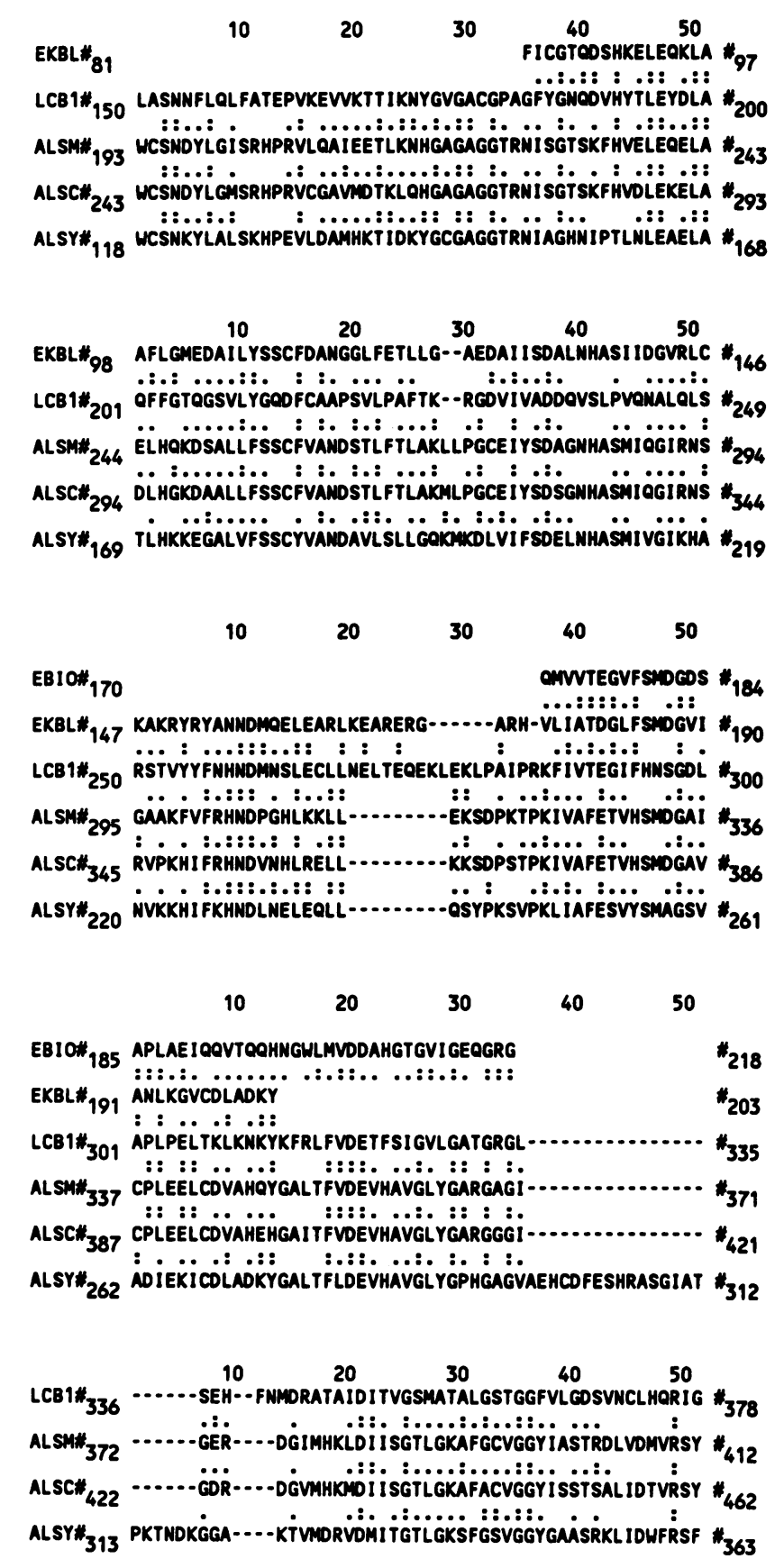

LCB1 $_{379}$ SHAYCFSACLPAYTVTSVSKVLKLMDSHNDAV

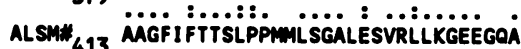

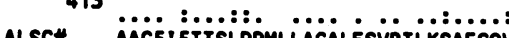

ALSC* $_{463}$ MGGFIFTTSLPPMLLÄGLLESVRTLKSAEGOV

ALSY* $_{364}$ APGFIFTTTLPPSVMAGATAM̈IRYQRCHIDLR

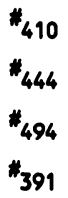


SERINE PALMTOYIRANSFERAS:

$\mathrm{CH}_{3}\left(\mathrm{CH}_{2}\right)_{14}-\mathrm{CO}-\mathrm{SCON}+\underset{m_{2}}{\mathrm{CONH}_{2} \mathrm{OH}} \stackrel{\text { PYRIDOXAL-P }}{\longrightarrow} \mathrm{CO}_{2}+\mathrm{CON}+\mathrm{CH}_{3}\left(\mathrm{CH}_{2}\right)_{14}-\mathrm{CO}_{-\mathrm{CH}_{2}-\mathrm{CH}_{2} \mathrm{OH}}$

5-AMNOLVUINC ACD SYNTHASE:

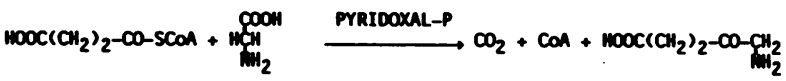

2-AMNO-3-KETOBUTMRATE LGASE:

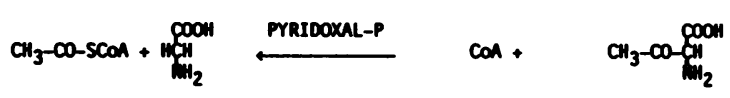

FIG. 7. Comparison of the reactions catalyzed by serine palmitoyltransferase, ALA synthase, and 2-amino-3-ketobutyrate CoA ligase.

ketobutyrate CoA ligase (Fig. 6). These two enzymes and serine palmitoyltransferase catalyze very similar chemical reactions, and all use the cofactor pyridoxal phosphate (Fig. 7). The similarity of the amino acid sequences and the reactions catalyzed by these three enzymes argues that the product of $L C B I$ is most likely serine palmitoyltransferase or a catalytic subunit of the enzyme, rather than a regulatory protein that regulates transcription of $L C B I$ or the activity of serine palmitoyltransferase.

ALA synthase catalyzes the first step in heme biosynthesis. Molecular cloning techniques have revealed the primary structure, regulation, and mechanisms of synthesis and transport of ALA synthase. For example, the nucleotide sequence of cDNAs encoding the human $(2)$, rat $(38,45)$, and chicken $(3,4,21)$ hepatic enzymes and the mouse (33) and chicken (31) erythroid isozymes along with the complete nucleotide sequences of ALA synthase genes from $S$. cerevisiae (HEMI) (41) and Bradyrhizobium japonicum (hemA) (24) have revealed structural similarities between the predicted primary sequences of these enzymes.

Because the first homology search with the entire LCB1 peptide could have missed proteins having small regions of amino acid similarity, a second homology search was performed with small, overlapping portions of LCB1. Two sets of contiguous 30-residue peptides were used in the second search, with one set overlapping the other by 15 residues. Many members of the two sets of peptides showed high sequence similarity to numerous proteins in the translated GENBANK data base by the FASTA algorithm: the ALA synthase similarities shown in Fig. 6 did not have particularly high similarity scores and would not have been seen as significant without further analysis. To identify statistically and biologically significant similarities, we searched for proteins having similarity to two or more of the 30-residue peptides, since this approach would identify larger regions of similarity. This strategy revealed the ALA synthase results shown in Fig. 6, and in addition, the similarities to the $E$. coli enzymes 2-amino-3-ketobutyrate CoA ligase (EKBL, Fig. 6) and biotin synthetase (EBIO, Fig. 6).

The similarity of the LCB1 protein to 2-amino-3-ketobutyrate CoA ligase seemed particularly significant since the ligase catalyzes a reaction (Fig. 7) that is very similar to those catalyzed by serine palmitoyltransferase and ALA synthase. In addition, the $E$. coli 2-amino-3-ketobutyrate CoA ligase uses pyridoxal phosphate as a cofactor (26), as do serine palmitoyltransferase and ALA synthase. 2-Amino-3- ketobutyrate CoA ligase catalyzes the second reaction in the threonine utilization pathway in both procaryotes (16) and eucaryotes (8).

The region of amino acid sequence similarity shown in Fig. 6 most likely defines the catalytic domain(s) of serine palmitoyltransferase, ALA synthase, and 2-amino-3-ketobutyrate CoA ligase. However, this suggestion awaits further analysis since the catalytic site(s) of none of these enzymes has been determined.

The similarity of the LCB1 peptide to biotin synthetase, the product of bioB, seems statistically significant because it covered a region of 49 amino acids (Fig. 6) in which $40.8 \%$ of the residues were identical and another $44.8 \%$ were similar. The functional significance of this similarity is not obvious since biotin synthetase catalyzes the addition of sulfur to $d$-dethiobiotin to yield biotin (see reference 27 for references) by a reaction not yet identified but seemingly different than that catalyzed by serine palmitoyltransferase.

What might be the function of the $\mathrm{N}$-terminal portion of LCB1, the portion that lacks similarity to those of other proteins? One possible function is to direct the protein to its proper cellular location. The mature form of serine palmitoyltransferase is found in the microsomal membrane fraction of animal cells, but its exact cellular location is unknown (25). Preliminary data (30) indicate that the $S$. cerevisiae serine palmitoyltransferase is a membrane-bound enzyme. The predicted LCB1 protein has two potential membranespanning helices (Fig. 5) that could tether the protein to the membrane. One of these helices, I in Fig. 5, is in the $\mathrm{N}$-terminal domain that shows no amino acid similarity to ALA synthase, so this region might target the protein to a specific membrane location.

Analysis of the $5^{\prime}$ noncoding region of $L C B 1$ revealed several putative TATA boxes, including TATTTAT at position -43 , AATATA at position -70 , TATAA at position -103 , and TAAAAAAAATT at position -140 . Since nothing is known about the regulation of $L C B 1$ or any other eucaryotic gene required for sphingolipid biosynthesis, mutational analysis of the $5^{\prime}$ upstream region may reveal the presence of novel cis-acting elements that regulate transcription.

We have described the initial characterization $L C B I$ and its predicted protein product. The gene most likely codes for serine palmitoyltransferase. An alternative explanation for our data would argue that $L C B 1$ is a regulatory gene whose product controls the synthesis or activity of serine palmitoyltransferase. While this possibility seems unlikely, further data will be needed to prove that $L C B 1$ codes for serine palmitoyltransferase or a subunit of the enzyme. Isolation of $L C B I$ has already proven to be useful by allowing the isolation of mutant yeast strains that lack detectable sphingolipids (9). The gene should be useful for a variety of other studies.

\section{ACKNOWLEDGMENTS}

This work was supported by NIH grants GM41302 and AI20600.

\section{REFERENCES}

1. Aronson, B. A., P. D. Ravnikar, and R. L. Somerville. 1988. Nucleotide sequence of the 2-amino-3-ketobutyrate coenzyme A ligase $(k b l)$ gene of $E$. coli. Nucleic Acids Res. 16:3586.

2. Bawden, M. J., I. A. Borthwick, H. M. Healy, C. P. Morris, B. K. May, and W. H. Elliott. 1987. Sequence of human 5-aminolevulinate synthase cDNA. Nucleic Acids Res. 15:8563.

3. Borthwick, I. A., G. Srivastava, J. D. Brooker, B. K. May, and W. H. Elliott. 1983. Purification of 5-aminovulinate synthase. 
Eur. J. Biochem. 129:615-620

4. Borthwick, I. A., G. Srivastava, A. R. Day, B. A. Pirola, M. A. Snoswell, B. K. May, and W. H. Elliott. 1985. Complete nucleotide sequence of hepatic 5-aminolevulinate synthase precursor. Eur. J. Biochem. 150:481-484.

5. Brennan, P. J., and D. M. Losel. 1978. Fungal lipids, p. 47-179. In A. H. Rose and P. G. Morris (ed.), Microbial physiology, vol. 17. Academic Press, Inc., New York

6. Carlson, M., and D. Botstein. 1982. Two differentially regulated mRNAs with different 5 ' ends encode secreted and intracellular forms of yeast invertase. Cell 28:145-154.

7. Chang, Y., and R. C. Dickson. 1988. Primary structure of the lactose permease gene from the yeast Kluyveromyces lactis. J. Biol. Chem. 263:16696-16703.

8. Dale, R. A. 1978. Catabolism of threonine in mammals by coupling of L-threonine 3-dehydrogenase with 2-amino-3-oxobutyrate-CoA ligase. Biochim. Biophys. Acta 544:496-503.

9. Dickson, R. C., G. B. Wells, A. Schmidt, and R. L. Lester. 1990. Isolation of mutant Saccharomyces cerevisiae strains that survive without sphingolipids. Mol. Cell. Biol. 10:2176-2181.

10. Eisenberg, D., E. Schwartz, M. Komaromy, and R. Wall. 1984. Analysis of membrane and surface protein sequences with the hydrophobic moment plot. J. Mol. Biol. 179:125-142.

11. Feinberg, A. P., and B. Vogelstein. 1983. A technique for radiolabeling DNA restriction endonuclease fragments to high specific activity. Anal. Biochem. 132:6-13.

12. Gornall, A. G., C. J. Bardwill, and M. M. David. 1949. Determination of serum proteins by means of the biuret reaction. $\mathrm{J}$. Biol. Chem. 177:751-756.

13. Hakomori, S.-I. 1983. Chemistry of sphingolipids, p. 1-150. In J. N. Kanfer and S. I. Hakomori (ed.), Sphingolipid biochemistry. Plenum Publishing Corp., New York.

14. Hakomori, S.-I. 1989. Aberrant glycosylation in tumors and tumor-associated carbohydrate antigens. Adv. Cancer Res. 52: 257-326.

15. Hannun, Y. A., and R. M. Bell. 1989. Function of sphingolipids and sphingolipid breakdown products in cellular regulation. Science 243:500-507.

16. Komatsubara, S., K. Murata, M. Kisumi, and I. Chibata. 1978. Threonine degredation in Serratia marcescens. J. Bacteriol. 135:318-323.

17. Kyte, J., and R. F. Doolittle. 1982. A simple method for displaying the hydropathic character of a protein. J. Mol. Biol. 157:105-132.

18. Laine, R. A., T. C.-Y. Hsieh, and R. L. Lester. 1980. Glycophosphoceramides from plants, p. 65. In C. C. Sweeley (ed.), Cell surface glycolipids, American Chemical Society Symposium Series 128. American Chemical Society, Washington, D.C.

19. Ledeen, R. W. 1989. Biosynthesis, metabolism, and biological effects of gangliosides, p. 43-83. In R. U. Margolis and R. K. Margolis (ed.), Neurobiology of glyconjugates. Plenum Publishing Corp., New York.

20. Lev, M., and A. F. Milford. 1973. The 3-ketodihydrosphingosine synthetase of Bacteroides melaninogenicus: induction by vitamin K. Arch. Biochem. Biophys. 157:500-508.

21. Maguire, D. J., A. R. Day, I. A. Borthwick, G. Srivastava, P. L. Wrigley, B. K. May, and W. H. Elliott. 1986. Nucleotide sequence of the chicken 5-aminolevulinate synthase gene. Nucleic Acids Res. 14:1379-1391.

22. Maniatis, T., E. F. Fritsch, and J. Sambrook. 1982. Molecular cloning: a laboratory manual, Cold Spring Harbor Laboratory, Cold Spring Harbor, N.Y.

23. Marshall, R. D. 1972. Glycoproteins. Annu. Rev. Biochem. 41:673-702.

24. McClung, C. R., J. E. Somerville, M. L. Guerinot, and B. K. Chelm. 1987. Structure of Bradyrhizobium japonicum hemA encoding 5-aminolevulinic acid synthase. Gene 54:133-139.

25. Merrill, A. H., Jr., D. W. Nixon, and R. D. Williams. 1985. Activities of serine palmitoyltransferase (3-keto-sphinganine synthase) in microsomes from different rat tissues. J. Lipid Res. 26:617-622.

26. Mukherjee, J. J., and E. E. Dekker. 1987. Purification, properties, and $\mathrm{N}$-terminal amino acid sequence of homogeneous
Escherichia coli 2-amino-3-ketobutyrate CoA Ligase, a pyridoxal phosphate-dependent enzyme. J. Biol. Chem. 262:1444114447

27. Otsuka, A. J., M. R. Buoncristiani, P. K. Howard, J. Flamm, C. Johnson, R. Yamamoto, K. Uchida, C. Cook, J. Ruppert, and J. Matsuzaki. 1988. The Escherichia coli biotin biosynthetic enzyme sequences predicted from the nucleotide sequence of the bio operon. J. Biol. Chem. 263:19577-19585.

28. Pearson, W. R., and D. J. Lipman. 1988. Improved tools for biological sequence comparison. Proc. Natl. Acad. Sci. USA 85:2444-2448.

29. Petko, L., and S. Lindquist. 1986 . Hsp26 is not required for growth at high temperature, nor for thermotolerance, spore development, or germination. Cell 45:885-894.

30. Pinto, W. J., G. B. Wells, A. C. Williams, K. A. Anderson, E. C. Teater, and R. L. Lester. 1986. Characterization of a S. cerevisiae mutant defective in inositol sphingolipid synthesis. Fed. Proc. 45:1826.

31. Riddle, R. D., M. Yamamoto, and J. D. Engel. 1989. Expression of $\delta$-aminolevulinate synthase in avian cells: separate genes encode erythroid-specific and nonspecific isozymes. Proc. Natl. Acad. Sci. USA 86:792-796.

32. Rose, M. D. 1987. Isolation of genes by complementation in yeast. Methods Enzymol. 152:481-504.

33. Schoenhaut, D. S., and P. J. Curtis. 1986. Nucleotide sequence of mouse 5-aminolevulinic acid synthase cDNA and expression of its gene in hepatic and erythroid tissues. Gene 48:55-63.

34. Sherman, F., G. R. Fink, and T. B. Hicks. 1987. Methods in yeast genetics: a laboratory course manual. Cold Spring Harbor Laboratory, Cold Spring Harbor, N.Y.

35. Sikorski, R. S., and P. Hieter. 1989. A system of shuttle vectors and yeast host strains designed for efficient manipulation of DNA in Saccharomyces cerevisiae. Genetics 122:19-27.

36. Smith, S. W., and R. L. Lester. 1974. Inositol phosphorylceramide, a novel substance and the chief member of a major group of yeast sphingolipids containing a single inositol phosphate. J. Biol. Chem. 249:3395-3405.

37. Snell, E. E., S. J. Di Mari, and R. N. Brady. 1970. Biosynthesis of sphingosine and dihydrosphingosine by cell-free systems from Hensenula cifferi. Chem. Phys. Lipids 5:116-138.

38. Srivastava, G., I. A. Borthwick, D. J. Maguire, C. J. Elferink, M. J. Bawden, J. F. B. Mercer, and B. K. May. 1988. Regulation of 5-aminolevulinate synthase mRNA in different rat tissues. $\mathrm{J}$. Biol. Chem. 263:5202-5209.

39. Steiner, S., S. Smith, C. J. Waechter, and R. L. Lester. 1969. Isolation and partial characterization of a major inositol-containing lipid in Baker's yeast, mannosyl-di-inositol, diphosphoryl-ceramide. Proc. Natl. Acad. Sci. USA 64:1042-1048.

40. Stoffel, W. 1970. Studies on the biosynthesis and degradation of sphingosine bases. Chem. Phys. Lipids 5:139-158.

41. Urban-Grimal, D., C. Wollard, T. Garnier, P. Dehoux, and R. Labbe-Boise. 1986. The nucleotide sequence of the HEMI gene and evidence for a precursor form of the mitochondrial 5-aminolevulinate synthase in Saccharomyces cerevisiae. Eur. J. Biochem. 156:511-519.

42. Wells, G. B., and R. L. Lester. 1983. The isolation and characterization of a mutant strain of Saccharomyces cerevisiae that requires a long chain base for growth and for synthesis of phosphosphingolipids. J. Biol. Chem. 258:10200-10203.

43. Williams, R. D., E. Wang, and A. H. Merrill. 1984. Enzymology of long-chain base synthesis by liver: characterization of serine palmitoyltransferase in rat liver microsomes. Arch. Biochem. Biophys. 228:282-291.

44. Wray, L. V., Jr., M. M. Witte, R. C. Dickson, and M. I. Riley. 1987. Characterization of a positive regulatory gene, $L A C$ 9, that controls induction of the lactose-galactose regulon of Kluyveromyces lactis: structural and functional relationships to GALA of Saccharomyces cerevisiae. Mol. Cell. Biol. 7:1111-1121.

45. Yamamoto, M., S. Kure, J. D. Engel, and K. Hiraga. 1988. Structure, turnover, and heme-mediated suppression of the level of mRNA encoding rat liver $\delta$-aminolevulinate synthase from the cytosol to the mitochondria and its regulation by hemin in rat liver. J. Biol. Chem. 263:15973-15979. 